\title{
Identification and genomic characterization of a novel species of feline anellovirus
}

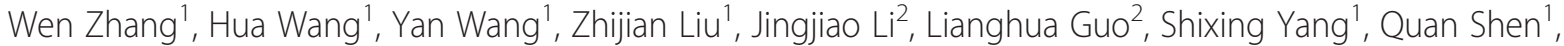 \\ Xiaoying Zhao', Li Cui ${ }^{2}$ and Xiuguo Hua ${ }^{2 *}$
}

\begin{abstract}
Here, a novel feline anellovirus strain (named FelineAV621 and GenBank no. KX262893) was detected in two cats with diarrhea. The complete genome of FelineAV621 is 2409 nt long with a G+C content of $56.67 \%$, including three open reading frames (ORFs). Phylogenetic analysis based on the amino acid sequence of the putative capsid protein (ORF1) indicated that FelineAV621 belonged to a novel anellovirus species inside a clade containing the seal anellovirus, canine TTVs, and porcine TTVs, but was distant from all the previous feline anelloviruses.
\end{abstract}

Keywords: Cat, Anellovirus, Complete genome, Viral metagenomics, Phylogenetic analysis

Abbreviations: BLAST, Basic local alignment search tool; ORF, Open reading frame; PCR, Polymerase chain reaction; SAV, Small anellovirus; TTMV, Torque teno mini virus, TTMDV, Torque teno midi virus; TTV, Torque teno virus

\section{Findings}

Anelloviruses belong to non-enveloped, circular, singlestranded DNA viruses with genome of 2.1-3.9 kb in length depending on the isolate analyzed [1]. Anelloviruses are subgrouped into Torque teno virus (TTV), Torque teno mini virus (TTMV), Torque teno midi virus (TTMDV) and small anellovirus (SAV) [2]. Although anelloviruses are widely prevalent in humans and animals and suspected to be associated with some diseases of humans [3-6] or animals [7-9], the etiological role of anelloviruses has not yet been clearly identified.

The first report of discovering feline anellovirus was published by Okamoto et al. [10] and subsequently reported in several other publications [11-14]. Phylogenetic analysis based on the amino acid sequences of ORF1 of feline anelloviruses available in GenBank revealed that these known feline anellovirus clustered together and were classified into two species, Torque teno felis virus 1 (FcTTV1) and Torque teno felis virus 2 (FcTTV2), belonging to the genus Etatorquevirus within the family Anelloviridae [13]. The aim of this study is to characterize the genome sequence of a divergent feline anellovirus which is present in the fecal and blood samples from domestic cats with diarrhea.

\footnotetext{
* Correspondence: hxg@sjtu.edu.cn

${ }^{2}$ School of Agriculture and Biology, Shanghai Jiaotong University, 800

Dongchuan Road, Shanghai 200240, China

Full list of author information is available at the end of the article
}

In a previous study, where viral metagenomic method was used to investigate the fecal virome of cats in a shelter in California, USA, several sequencing reads showed amino acid similarity to viruses in the family Anelloviridae [14]. In this study, nested primers designed based on a $621 \mathrm{bp}$ contig assembled by geneious software from the data of that study [14] are used to investigate whether this anellovirus is present in cat population in China. The primers used here are Anel621FO (5' - ACTTCCTCCT GGTCGGGCGT-3') and Anel621RO (5'- TGGGGAGG GGTTGATGCCCA-3') for the 1st round PCR, and Anel621FI (5'- GGCTCTCGTGCGGTTTGGGA-3') and Anel621RI (5' - TCCCGTCGTCCCACCACCAT-3') for the 2 nd round PCR. The PCR product size of the 2 nd round PCR is about $215 \mathrm{bp}$. PCR screening was performed to detect the anellovirus-like sequence in a total 22 samples, including 11 blood and 11 fecal samples collected from 11 cats with diarrhea from Jan. 2014 to Dec. 2015. Results indicated that two blood samples and one fecal samples were positive, including one cat positive both in blood and fecal samples, and one positive blood sample. The specific DNA band was T-A cloned and sequencing result indicated that the two sequences from blood and fecal samples of the same cat were identical and had one nucleotide difference from the sequence from the other blood sample in the present study. Sequence analysis indicated that the two sequences from cats in the present study shared $97.7 \%$ sequence identity with the 


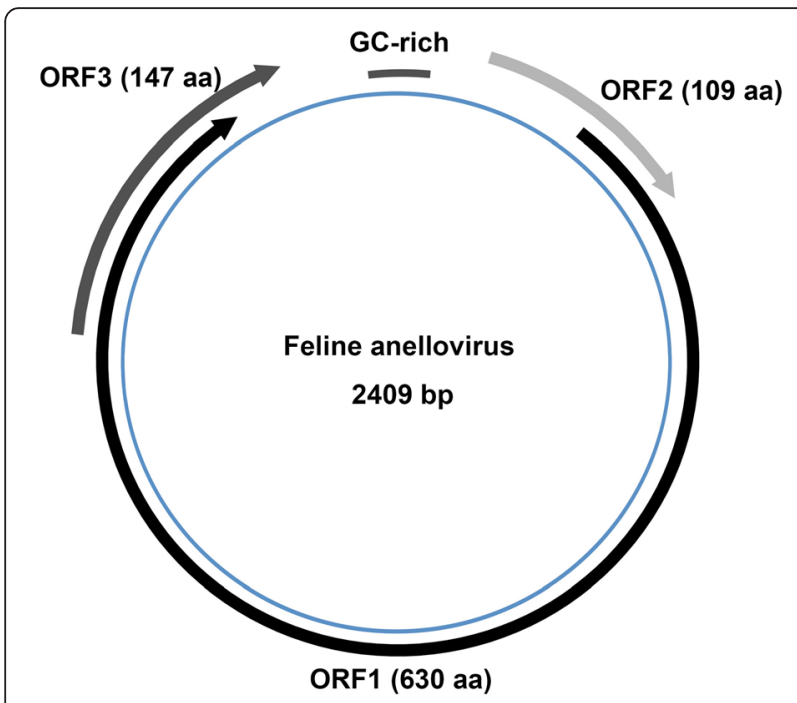

Fig. 1 Genomic organization of the feline anellovirus (named FelineAV621 and GenBank no. KX262893) sequences from the California cats [14], with five different nt over the 215 bp sequence fragment.

To sequence the entire genome of the feline anellovirus, the viral nucleic acid was extracted form one of the cat the blood samples. Circular viral DNA molecules were preferentially amplified by rolling circle amplification (RCA) using random hexamer primers and the Illustra TempliPhi amplification kit (GE Healthcare). Outwardly pointing specific PCR primers FelAVF (5'ATGCTCAACACCACAAACGC-3') and FelAVR (5'TGTAATCCCAAACCGCACGA-3') were designed from the viral metagenomic sequence [14] and then used to amplify the small circular genomes by inverse PCR. The PCR products were then sequenced by primer walking and Sanger sequencing.

Sequencing results indicated that the complete genome of the feline anellovirus strain (named FelineAV621 and GenBank no. KX262893) was 2409 nt long, with a $\mathrm{G}+\mathrm{C}$ content of $56.67 \%$. The genome organization of FelineAV621 is consistent with that of other anelloviruses,

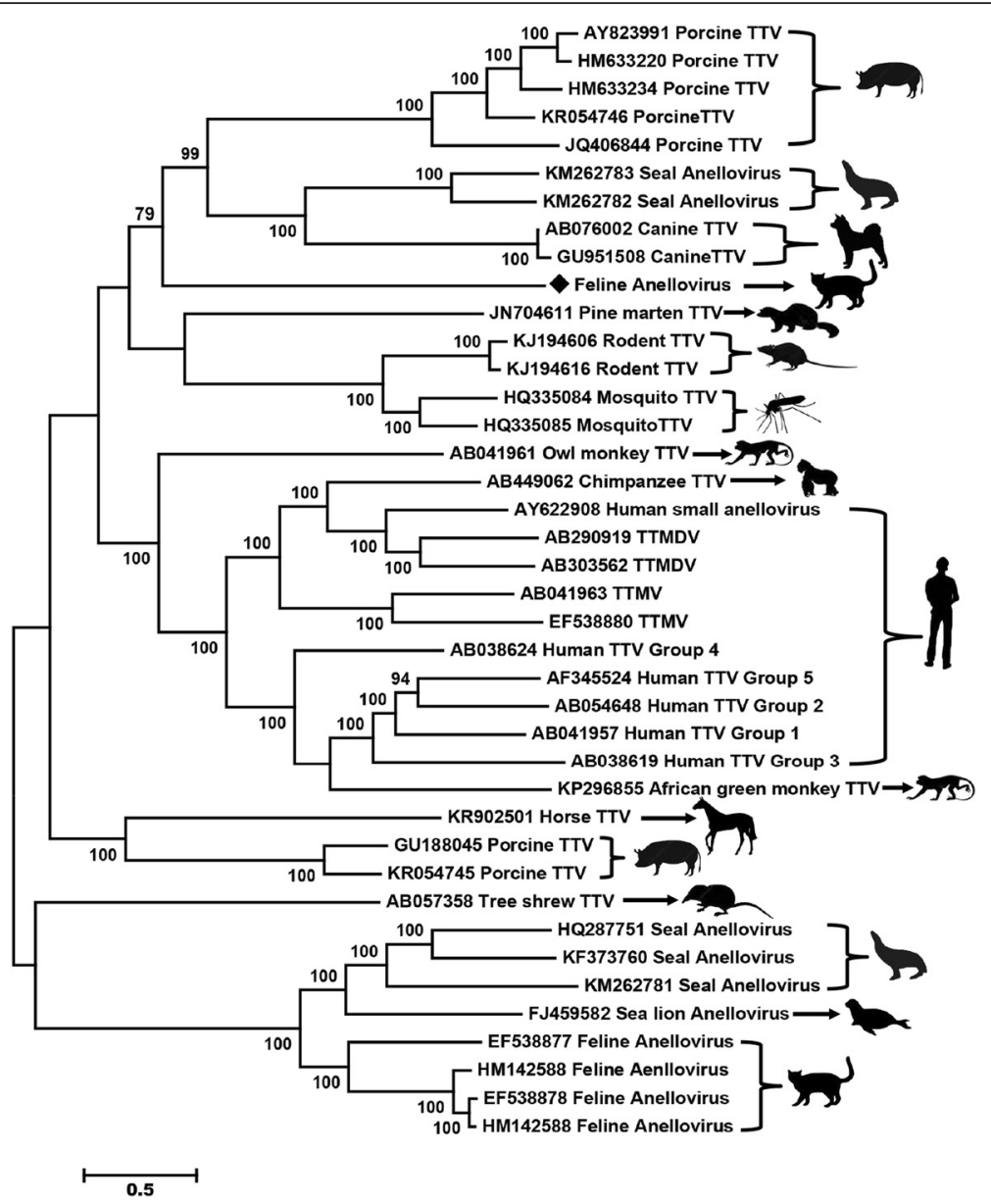

Fig. 2 Phylogenetic tree based on the full-length amino acid sequence of ORF1 depicting relationships among the members of the family Anelloviridae. The newly discovered feline anellovirus is indicated by a diamond shape. The host is indicated in animal profiles and GenBank accession numbers are shown 
containing three open reading frames (ORFs) (Fig. 1). The ORF1, the largest ORF, does not display any significant nucleotide identity to any virus sequence using BLASTn in GenBank, but shares low identity with a seal anellovirus (GenBank no.: KM262782) (30.2\% identity) [15] and a pine marten torque teno virus (GenBank no.: JN704611) (29.4 \% identity) [16] based on the amino acid sequence of ORF1. ORF1 encodes a 630 amino acid long putative capsid protein which presents three potential glycosylation sites; no signal peptides were identified. As in other anelloviruses, the capsid protein is arginine rich in its $\mathrm{N}$ terminus, with 31 arginine residues in the first 60 amino acids (51.67 \%). ORF2 encodes 109 amino acids and shares $29-40 \%$ amino acid sequence identities with several ORF2 proteins of anelloviruses in GenBank. ORF3 encodes147 amino acids and shares no amino acid sequence identity with proteins in GenBank.

To determine the relationship of FelineAV621 to other anelloviruses, phylogenetic analysis was performed based on the ORF1 amino acid sequences of FelineAV621, its best BLASTp matches in GenBank and the representative members of related viruses. Sequence alignment was performed using CLUSTALW with the default settings. A phylogenetic tree with 1000 bootstrap resamples of the alignment data sets was generated using the maximum likelihood method based on Jones-TaylorThornton (JTT) model in MEGA5.0. The anellovirus tree topology was consistent with results of earlier studies $[1,9,17]$ and revealed that FelineAV621 is a novel anellovirus species that belongs to a clade that contains the seal anellovirus, canine TTVs, and porcine TTVs, but distant from the previous four feline anelloviruses (Fig. 2), suggesting cat can carry at least two different species of anelloviruses.

Taken together, a novel species of feline anellovirus was detected in two cats with diarrhea, including both blood and fecal samples of one cat and the blood sample of the other one. The complete genome of this novel anellovirus was sequenced and characterized from one of the blood sample. Phylogenetic analysis revealed that this feline anellovirus belonged to a novel anellovirus species which is significantly divergent from the previous feline anellovirus strains. Due to the limited sample number and lacking serologic evidence in the present study, whether FelineAV621 can really infect cats and cause diarrhea will require further epidemiologic study based on a larger sample size and testing cat sera for specific antibodies.

\section{Funding}

This work was partly supported by the National Natural Science Foundation of China No. 31302107 and 31270186, the Natural Science Foundation of Jiangsu Province No. BK20130542, BK20140537, and BK20130502, and China Postdoctoral Special Foundation No. 2015 T80503.

\section{Authors' contributions}

WZ and XH conceived the study. WZ and HW performed all the experiments. WZ wrote the paper. All authors read and approved the final manuscript.

\section{Competing interests}

The authors declare that they have no competing interests.

\section{Author details}

${ }^{1}$ School of Medicine, Jiangsu University, 301 Xuefu Road, Zhenjiang, Jiangsu 212013, People's Republic of China. ${ }^{2}$ School of Agriculture and Biology,

Shanghai Jiaotong University, 800 Dongchuan Road, Shanghai 200240, China.

Received: 9 June 2016 Accepted: 15 August 2016

Published online: 27 August 2016

\section{References}

1. Biagini P. Classification of TTV and related viruses (anelloviruses). Curr Top Microbiol Immunol. 2009;331:21-33.

2. Bernardin F, Operskalski E, Busch M, Delwart E. Transfusion transmission of highly prevalent commensal human viruses. Transfusion. 2010;50:2474-83.

3. Tajiri H, Tanaka T, Sawada A, Etani Y, Kozaiwa K, Mushiake S, Mishiro S. Three cases with $T$ virus infection and idiopathic neonatal hepatitis. Intervirology. 2001:44:364-9.

4. Gergely P, Perl A, Poór G. Possible pathogenic nature of the recently discovered TT virus: does it play a role in autoimmune rheumatic diseases? Autoimmun Rev. 2006;6:5-9.

5. Ishimura M, Ohga S, Ichiyama M, Kusuhara K, Takada H, Hara T, Takahashi M, Okamoto $\mathrm{H}$. Hepatitis-associated aplastic anemia during a primary infection of genotype 1a torque teno virus. Eur J Pediatr. 2010;169:899-902.

6. Miyamoto M, Takahashi H, Sakata I, Adachi Y. Hepatitis-associated aplastic anemia and transfusion-transmitted virus infection. Intern Med. 2000;39:1068-70.

7. Krakowka S, Hartunian C, Hamberg A, Shoup D, Rings M, Zhang Y, Allan G, Ellis JA. Evaluation of induction of porcine dermatitis and nephropathy syndrome in gnotobiotic pigs with negative results for porcine circovirus type 2. Am J Vet Res. 2008;69:1615-22.

8. Teixeira TF, Cibulski SP, dos Santos HF, Wendlant A de Sales Lima FE, Schmidt C, Franco AC, Roehe PM. Torque teno sus virus 1 (TTSuV1) and 2 (TTSuV2) viral loads in serum of postweaning multisystemic wasting syndrome (PMWS)-affected and healthy pigs in Brazil. Res Vet Sci. 2015; 101:38-41.

9. Li L, Giannitti F, Low J, Keyes C, Ullmann LS, Deng X, Aleman M, Pesavento PA, Pusterla N, Delwart E. Exploring the virome of diseased horses. J Gen Virol. 2015:96:2721-33.

10. Okamoto H, Takahashi M, Nishizawa T, Tawara A, Fukai K, Muramatsu U, Naito Y, Yoshikawa A. Genomic characterization of TT viruses (TTVs) in pigs, cats and dogs and their relatedness with species-specific TTVs in primates and tupaias. J Gen Virol. 2002;83(Pt 6):1291-7.

11. Zhu CX, Shan TL, Cui L, Luo XN, Liu ZJ, Tang SD, Liu ZW, Yuan CL, Lan DL, Zhao W, Hua XG. Molecular detection and sequence analysis of feline Torque teno virus (TTV) in China. Virus Res. 2011;156:13-6.

12. Biagini $P$, Uch R, Belhouchet $M$, Attoui H, Cantaloube J-F, Brisbarre N, de Micco P. Circular genomes related to anelloviruses identified in human and animal samples by using a combined rolling-circle amplification/sequenceindependent single primer amplification approach. J Gen Virol. 2007;88(Pt 10):2696-701.

13. Jarošová V, Hrazdilová K, Filipejová Z, Schánilec P, Celer V. Whole genome sequencing and phylogenetic analysis of feline anelloviruses. Infect Genet Evol. 2015;32:130-4.

14. Zhang W, Li L, Deng X, Kapusinszky B, Pesavento PA, Delwart E. Faecal virome of cats in an animal shelter. J Gen Virol. 2014;95(Pt 11):2553-64.

15. Bodewes R, Contreras GJS, García AR, Hapsari R, van de Bildt MWG, Kuiken T, Osterhaus ADME. Identification of DNA sequences that imply a novel gammaherpesvirus in seals. J Gen Virol. 2015;96(Pt 5):1109-14.

16. Van den Brand JMA, van Leeuwen M, Schapendonk CM, Simon JH, Haagmans BL, Osterhaus ADME, Smits SL. Metagenomic analysis of the viral flora of pine marten and European badger feces. J Virol. 2012;86:2360-5.

17. Jones MS, Kapoor A, Lukashov W, Simmonds P, Hecht F, Delwart E. New DNA viruses identified in patients with acute viral infection syndrome. J Virol. 2005;79:8230-6. 